\title{
Self-reported Hypoglycaemic Events in 2430 Patients with Insulin-treated Diabetes in the German Sub-population of the HAT Study
}

\section{(ㄷ)(우우 $\Theta$}

\author{
Authors \\ Werner Kern¹, Andreas Holstein ${ }^{2}$, Christian Moenninghoff ${ }^{3}$, Joachim Kienhöfer ${ }^{3}$, Matthias RiedI ${ }^{4}$, Bernhard Kulzer ${ }^{5}$
}

Affiliations

1 Endokrinologikum, Ulm, Germany

2 Department of Medicine, Lippe-Detmold Hospital, Detmold, Germany

3 Novo Nordisk Pharma GmbH, Mainz, Germany

4 Medicum, Hamburg MVZ GmbH, Germany

5 Research Institute of the Diabetes Academy Mergentheim, Bad Mergentheim, Germany

Key words

hypoglycaemia, real-world, severe, nocturnal, Germany

received 30.12 .2016

revised 24.05.2017

accepted 29.05.2017

\section{Bibliography}

DOI https://doi.org/10.1055/s-0043-112350

Published online: 27.7.2017

Exp Clin Endocrinol Diabetes 2017; 125: 592-597

(C) J. A. Barth Verlag in Georg Thieme Verlag KG Stuttgart .

New York

ISSN 0947-7349

\section{Correspondence}

Werner Kern, MD

Endokrinologikum Ulm

Keltergasse 1

$89073 \mathrm{Ulm}$

Germany

Tel.: + 49/731/938088-0

werner.kern@endokrinologikum.com

\begin{abstract}
Data concerning true hypoglycaemic incidence in insulin-treated patients with diabetes in real-world clinical practice are lacking in Germany. The aim of this analysis was to determine the incidence of hypoglycaemia experienced by the German cohort of patients enrolled in the global Hypoglycaemia Assessment Tool (HAT) study. This was a non-interventional, 6-month retrospective and 4-week prospective study using self-assessment questionnaires and patient diaries assessing patients aged $\geq 18$ years in Germany, with type 1 diabetes (T1D) $(n=811)$ or type 2 diabetes (T2D) $(n=1619)$ treated with insulin for $>12$ months. The primary endpoint was the percentage of patients experiencing $\geq 1$ hypoglycaemic event during the prospective observational period ( 4 weeks after baseline). Predictive and continuous factors (such as age, gender, duration of insulin use and $\mathrm{HbA}_{1 \mathrm{c}}$ ) contributing to hypoglycaemia risk were explored.

During the prospective period, at least one hypoglycaemic event was reported by $81.3 \%$ of patients with T1D and $39.7 \%$ of patients with T2D, indicating that hypoglycaemia is a common acute complication among patients with insulin-treated diabetes. Severe hypoglycaemia was reported by $9.1 \%$ of patients with T1D and $5.4 \%$ of patients with T2D. Higher rates of any and severe hypoglycaemia were reported prospectively than retrospectively, regardless of diabetes type, indicating that patients retrospectively under-report hypoglycaemia. Prospective rates (events per patient-year) of any, nocturnal and severe hypoglycaemia were 80.3, 9.9 and 3.0 for T1D and 15.6, 2.4 and 1.1 for T2D, respectively. Given the potential for recall bias in retrospective reporting, this prospective assessment of hypoglycaemia appears more reliable than retrospective assessment.
\end{abstract}

Trial number: NCT01696266

\section{Introduction}

The prevalence of diabetes is increasing worldwide. The International Diabetes Federation (IDF) estimates there were 415 million people living with diabetes in 2015, of which approximately 6.5 million were in Germany [1]. The key clinical goal in the management of diabetes is to maintain normal blood glucose levels, often by treating with insulin therapy. Insulin is commonly used in the treatment of type 1 diabetes (T1D), and less commonly used in type 2 diabetes (T2D).

Hypoglycaemia is a common side effect of insulin therapy and remains the main barrier to effective glycaemic control [2]. In particular, recurrent episodes of hypoglycaemia may result in poor adherence to treatment. Severe hypoglycaemia (irrespective of diabetes type) is associated with poor prognosis, and if untreated can 
result in coma, seizures, or death [3]. Severe hypoglycaemia may also increase the risk of cardiovascular complications and all-cause mortality [4, 5]. Hypoglycaemia is associated with reduced wellbeing and health-related quality of life, and increased health care costs [6]. Furthermore, hypoglycaemia can impact negatively on cognitive function [7].

Although data are available for rates of hypoglycaemia from randomised controlled trials (RCTs), evidence from real-world clinical practice is limited, meaning the true frequency of hypoglycaemia is difficult to determine. International prospective studies have reported incidences ranging from 3.9-7.2 events/month for patients with T1D [8-10] and 0.76-3.7 events/month for patients with T2D [8-13]. Differences between reporting methods of retrospective and prospective data may exist and there may be a lack of consistency in definition of hypoglycaemia. Patients with T1D have been shown to be able to recall incidences of severe hypoglycaemia for up to a year after their occurrence, but there is no evidence on the reliability of recall for the incidence of non-severe hypoglycaemia [14]. Hypoglycaemia rates have been shown to be under-reported in RCTs compared with observational studies [15]. Thus, real-world data are needed to evaluate the true incidence of hypoglycaemia in clinical practice.

Data on the real-world rate of hypoglycaemia in Germany are limited. Rates of non-severe hypoglycaemia from a small real-world study in Germany were reported in 2014 and suggested that the burden of hypoglycaemia had previously been underestimated [16]. The wide range of reported hypoglycaemic incidences highlights the need for additional data to determine factors related to hypoglycaemia risk.

The global Hypoglycaemia Assessment Tool (HAT) study was designed to determine the incidence of hypoglycaemia in a global insulin-treated patient population. The primary aim of this study was to determine the percentage of patients experiencing at least one hypoglycaemic event during the observational period among insulin-treated patients with T1D and T2D. This analysis presents data from the German cohort of the global HAT population.

\section{Methods}

\section{Study design}

The current analysis evaluates data from a German cohort of insulin-treated patients with diabetes enrolled in the HAT study, a noninterventional, multicentre, 6-month retrospective and 4-week prospective study of hypoglycaemic events in patients with T1D and T2D. The study design and patient population of the global HAT study have been previously described [5].

The study protocol and assessments were conducted in accordance with the Declaration of Helsinki [17] and the International Conference on Harmonisation Guidelines for Good Clinical Practice [18], and approved by the German Regulatory agency BfArM: No. 1662. All study materials were translated into local languages, and data obtained were translated back into English for analysis. Written informed consent was given by all participants.

\section{Study population}

Consecutive eligible patients were enrolled during routinely scheduled clinical consultations with their healthcare practitioners across 176 sites in Germany. Patients were eligible for study inclusion if they had T1D or T2D, were treated with any insulin for $>12$ months, were $\geq 18$ years of age at the time of the survey and had given informed consent to participate in the survey. Patients were excluded from the study if they were non-ambulatory, illiterate or otherwise unable to complete the written survey.

\section{Study endpoints}

The primary endpoint was the percentage of insulin-treated patients with T1D or T2D experiencing at least one hypoglycaemic event during the observational period. Secondary endpoints included hypoglycaemia incidence rates and the relationship between hypoglycaemia and predictive or continuous factors such as age, gender, duration of insulin use, or glycated haemoglobin $\left(\mathrm{HbA}_{1 \mathrm{c}}\right.$ ) (including proportion of patients with $\mathrm{HbA}_{1 \mathrm{c}}<7.0 \%$ [53 mmol $/ \mathrm{mol}$ ], $7.0-9.0 \%$ and $>9.0 \%$ [75 mmol $/ \mathrm{mol}]$ ), fear of hypoglycaemia (continuous on scale of 0 to 10 , where 0 is not afraid at all and 10 is absolutely terrified).

\section{Assessments}

Assessment was by a two-part self-assessment questionnaire (SAQ) consisting of a retrospective cross-sectional evaluation (Part 1), and a prospective observational evaluation (Part 2). Part 1 (baseline) was used to record baseline demographic and treatment information, knowledge of hypoglycaemia, hypoglycaemia unawareness, perceptions of hypoglycaemia, as well as the history of severe hypoglycaemia over the previous 6 months and symptomatic hypoglycaemia over the previous 4 weeks. Part 2 ( 4 weeks after baseline) was used to record history of both severe and symptomatic hypoglycaemia in the 4 weeks following study entry.

At baseline, patients were issued with a diary to assist recall and to record hypoglycaemic events, the effect of hypoglycaemia on productivity, and healthcare utilisation and productivity over the 4 weeks following study entry. Paired responses to the Part 1 and Part 2 SAQs were used to estimate the frequency of under-reporting of hypoglycaemia.

Data are presented from the full analysis set (FAS; patients completing Part 1) and completers' analysis set (CAS; patients completing both Part 1 and 2).

Patients were able to report hypoglycaemia by using symptoms alone, blood glucose measurement $(<3.1 \mathrm{mmol} / \mathrm{L}$ ) alone, or both symptoms and blood glucose measurements. Any reported selfmeasured glucose values $<3.1 \mathrm{mmol} / \mathrm{L}$ were considered to be 'true' hypoglycaemia.

\section{Hypoglycaemia classification}

Hypoglycaemia was defined and classified as follows: severe hypoglycaemia, a hypoglycaemic event requiring third-party assistance (consistent with the American Diabetes Association definition [3]); non-severe hypoglycaemia, an event managed by the patient alone; any hypoglycaemic event, the sum of severe and non-severe hypoglycaemia; nocturnal hypoglycaemia; hypoglycaemia occurring between midnight and 06:00 h. 
The combined measure of any hypoglycaemia was derived from both the diary and SAQ entries.

\section{Statistical analysis}

All statistical tests were two-sided and regarded as exploratory. Statistical significance was set at $p<0.05$ and no adjustments were made for multiple comparisons. The percentage of patients experiencing at least one hypoglycaemic event during the observation period was calculated, together with the $95 \%$ confidence interval (Cl) for this percentage. A binomial distribution was assumed. The relationship between incidence of hypoglycaemia and various factors was explored using negative binomial regression models stratified by country and specifying a log-transformed exposure time offset term. The majority of analyses were descriptive in nature, hence imputation of missing data was not performed. Data are presented as mean \pm standard deviation (SD) unless otherwise specified.

\section{Results}

\section{Patient characteristics}

Overall, 2430 patients completed the part 1 SAQ, 2056 (84.6\%) completed the part 2 SAQ and 2044 (84.1\%) patients completed patient diaries. Of the 2430 patients who completed part 1 of the SAQ, 811 had T1D and 1619 had T2D. Patient baseline characteristics are given in > Table 1. Overall, $53 \%$ of patients were male, and patients with T1D were younger than those with T2D (47.8 years vs. 66.3 years, respectively). The mean duration of diabetes was longer in patients with T1D (19.9 years) than those with T2D (15.4 years). $\mathrm{HbA}_{1 \mathrm{c}}$ levels were similar in patients with T1D and T2D [7.6\% (59.6 mmol $/ \mathrm{mol})$ vs. $7.5 \%$ (58.3 mmol $/ \mathrm{mol})$, respectively]. The most widely used methods for treating diabetes were shortacting and long-acting insulin. A greater proportion of patients with T1D than T2D reported that they had ever experienced hypoglycaemia (97.1\% vs. $76.3 \%$, respectively).

\section{Hypoglycaemic frequency}

A greater proportion of patients with T1D than T2D reported any hypoglycaemic event in the prospective period ( $81.3 \%$ vs. $39.7 \%$, respectively). The prevalence and incidence of any, nocturnal and severe hypoglycaemia are shown in $>$ Table 2 . Rates (estimated number of events per patient-year [PPY]) of any and severe hypoglycaemia were higher in the prospective period than in the retrospective period. Higher rates were reported for T1D than T2D for the occurrence of any hypoglycaemic event during the prospective and retrospective periods. The difference in incidence rate ratio between the retrospective ( 4 weeks before baseline) and prospective (4 weeks after baseline) periods for T1D and T2D are shown in > Fig. 1.

The incidence rate ratio (IRR) for any hypoglycaemic event in the prospective versus retrospective periods was $1.62(95 \% \mathrm{Cl} 1.42$; 1.85; $\mathrm{p}<0.001$ ) for T1D and 1.39 (95\% Cl 1.23; 1.56; $\mathrm{p}<0.001)$ for T2D. Overall, $64.2 \%$ of all patients identified hypoglycaemia by symptoms and using blood glucose measurements
- Table 1 Characteristics of patient population.

\begin{tabular}{|c|c|c|}
\hline Characteristic & T1D $(n=811)$ & T2D $(n=1619)$ \\
\hline $\begin{array}{l}\text { Number of patients who } \\
\text { completed part } 2 \text { SAQ, } \mathrm{n}(\%)\end{array}$ & $641(79.0)$ & $1415(87.4)$ \\
\hline Sex male/female, \% & $51 / 49$ & $54 / 46$ \\
\hline Mean age, years (SD) & $47.8(14.7)$ & $66.3(10.5)$ \\
\hline Duration of diabetes, years (SD) & $19.9(12.8)$ & $15.4(8.8)$ \\
\hline $\mathrm{HbA}_{1 \mathrm{c}}, \mathrm{mmol} / \mathrm{mol}(\mathrm{SD})$ & $59.6(13.8)$ & $58.3(13.6)$ \\
\hline $\mathrm{HbA}_{1 \mathrm{c}}, \% *$ & 7.6 & 7.5 \\
\hline \multicolumn{3}{|c|}{ Method of diabetes treatment, n (\%) } \\
\hline Short-acting insulin & $671(82.9)$ & $1237(77.0)$ \\
\hline Long-acting insulin & $517(63.9)$ & $1348(83.9)$ \\
\hline Mixed insulin & $7(0.9)$ & $129(8.0)$ \\
\hline Insulin pump & $228(28.2)$ & $15(0.9)$ \\
\hline Oral antidiabetes treatments & $17(2.1)$ & 449 (27.9) \\
\hline $\begin{array}{l}\text { Injectable antidiabetes } \\
\text { treatments excluding insulin }\end{array}$ & $0(0.0)$ & $27(1.7)$ \\
\hline \multicolumn{3}{|l|}{ Checks blood sugar levels, n (\%) } \\
\hline Yes & $806(99.4)$ & $1597(98.8)$ \\
\hline No & $5(0.6)$ & $20(1.2)$ \\
\hline \multicolumn{3}{|l|}{ Experienced hypoglycaemia, n (\%) } \\
\hline Yes & $780(97.1)$ & $1224(76.3)$ \\
\hline No & $13(1.6)$ & $322(20.1)$ \\
\hline Not sure & $10(1.2)$ & $58(3.6)$ \\
\hline \multicolumn{3}{|c|}{$\begin{array}{l}\text { * Calculated, not measured. Full analysis set; SAQ, self-assessment } \\
\text { questionnaire; SD, standard deviation; T1D, type } 1 \text { diabetes; T2D, } \\
\text { type } 2 \text { diabetes }\end{array}$} \\
\hline
\end{tabular}

\section{Associations between hypoglycaemia and continu- ous or predictor variables}

The proportions of patients with T1D and T2D experiencing hypoglycaemia, stratified by $\mathrm{HbA}_{1 \mathrm{c}}$ level, is shown in > Fig. 2 . $\mathrm{HbA}_{1 \mathrm{c}}$ levels were weakly associated with any hypoglycaemia (IRR 0.99; $95 \%$ $\mathrm{Cl} 0.99 ; 0.99)$. A weak correlation was also seen with hypoglycaemia and age (IRR 0.98; 95 \% Cl 0.98-0.99). Overall, hypoglycaemia was more likely to be reported by females than males (IRR 1.30; $95 \% \mathrm{Cl} 1.15 ; 1.46)$. Hypoglycaemic incidence rates increased with each quartile for duration of insulin use during the 4 weeks before and the 4 weeks after baseline in patients with T1D and T2D.

\section{Patient perspectives and actions relating to hypoglycaemia}

Patient perspectives with respect to knowledge, definition of hypoglycaemia and fear of hypoglycaemia are shown in $>$ Table 3. A slightly higher percentage of patients with T1D than T2D knew what hypoglycaemia was before reading the definition provided in the SAQ ( $97.3 \%$ vs. $87.3 \%$, respectively). There were no notable differences between patients with T1D and T2D for fear of hypoglycaemia with a mean (SD) score of 4.1 (2.75) for patients with T1D and 3.2 (2.88) for patients with T2D. Patients with T1D were more likely than those with T2D to increase glucose monitoring following a hypoglycaemic event (74.3\% T1D vs. $49.6 \%$ T2D).

The proportion of patients with hypoglycaemic events requiring hospital admission in the prospective period was $2.8 \%$ for T1D and $0.7 \%$ for T2D. The estimated annual incidence rate of hypogly- 
- Table 2 Hypoglycaemia incidence and prevalence in retrospective and prospective periods.

\begin{tabular}{|c|c|c|c|c|}
\hline & \multicolumn{2}{|c|}{ Retrospective } & \multicolumn{2}{|c|}{ Prospective $^{\dagger}$} \\
\hline & T1D & T2D & T1D $(n=648)$ & T2D $(n=1423)$ \\
\hline \multicolumn{5}{|c|}{ Hypoglycaemia prevalence, n (\% of patients) } \\
\hline Any (4 weeks) & $671(82.8)$ & $727(45.3)$ & $527(81.3)$ & $564(39.7)$ \\
\hline Nocturnal (4 weeks) & $342(43.2)$ & $257(16.5)$ & $225(37.3)$ & $159(12.2)$ \\
\hline Severe & $188(23.5)^{*}$ & $158(9.9)^{*}$ & $59(9.1)$ & $77(5.4)$ \\
\hline \multicolumn{5}{|c|}{ Hypoglycaemia incidence, events per patient-year } \\
\hline Any (4 weeks) & 50.0 & 11.2 & 80.3 & 15.6 \\
\hline Nocturnal (4 weeks) & 14.4 & 3.6 & 9.9 & 2.5 \\
\hline Severe & $2.1^{*}$ & $0.4^{*}$ & 3.0 & 1.1 \\
\hline
\end{tabular}

baseline. Incidence and prevalence calculated from full analysis set; T1D, type 1 diabetes; T2D, type 2 diabetes

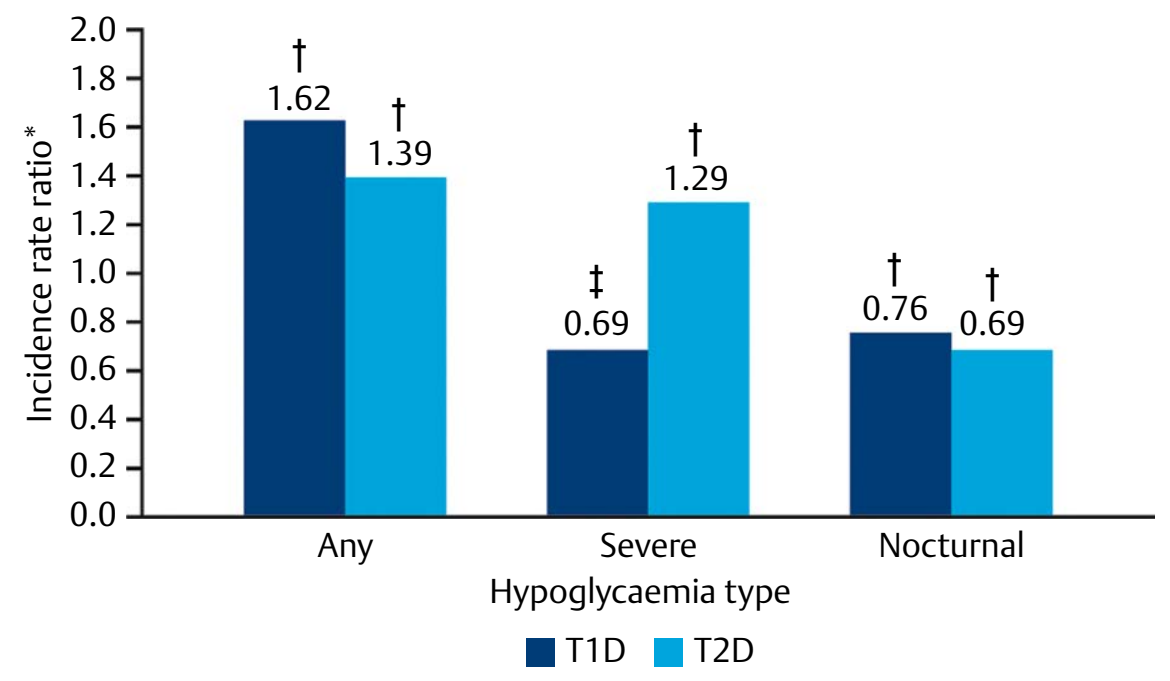

- Fig. 1 Difference in reported incidence of hypoglycaemia between retrospective and prospective groups by diabetes type.

* 4 weeks before $/ 4$ weeks after baseline; ${ }^{\dagger} p<0.001 ;{ }^{\ddagger} p=0.071$. T1D, type 1 diabetes; T2D, type 2 diabetes.

caemic events requiring hospitalisation in the prospective period was 0.236 [95\% Cl 0.118; 0.42] for T1D and 0.097 [95\% Cl 0.046; $0.178]$ for T2D.

\section{Discussion and Conclusion}

This analysis presents data from the German cohort of the HAT study. The global HAT study represents one of the largest cohorts of insulin-treated patients with diabetes to report data on hypoglycaemia, and has provided important information on the incidence of hypoglycaemia in patients with T1D and T2D, showing that the rates are higher than many previous estimates [17]. Consistent with the overall findings of the HAT study, rates of hypoglycaemia were higher in the current analysis than reported in other previous studies [8-10,12,13]. The overall rates of hypoglycaemic incidence (events PPY) in Germany were higher in the prospective period in patients with T1D than that reported in the HAT study (80.3 vs. 73.3 events PPY, respectively) [17], whilst the overall rates for patients with T2D were slightly lower than that reported in the
HAT study (15.6 vs. 19.3 events PPY, respectively). Patients in the German cohort were older, had a longer duration of diabetes and lower $\mathrm{HbA}_{1 \mathrm{c}}$ compared with the global HAT cohort.

Importantly for clinical practice, a weak association with $\mathrm{HbA}_{1 \mathrm{c}}$ and hypoglycaemia was observed, suggesting that treatment may need to be individualised depending on how well patients respond.

Higher rates of any hypoglycaemic event were reported prospectively versus retrospectively, indicating patients may retrospectively under-report hypoglycaemic incidences. Prospective assessment of hypoglycaemia appears to be more reliable than retrospective recall, possibly as a consequence of recall bias.

\section{Cost of diabetes medicines in Germany}

In addition to the clinical burden, diabetes also has a significant impact on the economics of diabetes healthcare. Severe hypoglycaemic events often result in emergency/ambulance calls and hospital treatment and incur substantial healthcare costs [19]. Koster et al. carried out an evaluation of healthcare expenditure and the direct cost of diabetes on the German healthcare system in 2010 [20]. 


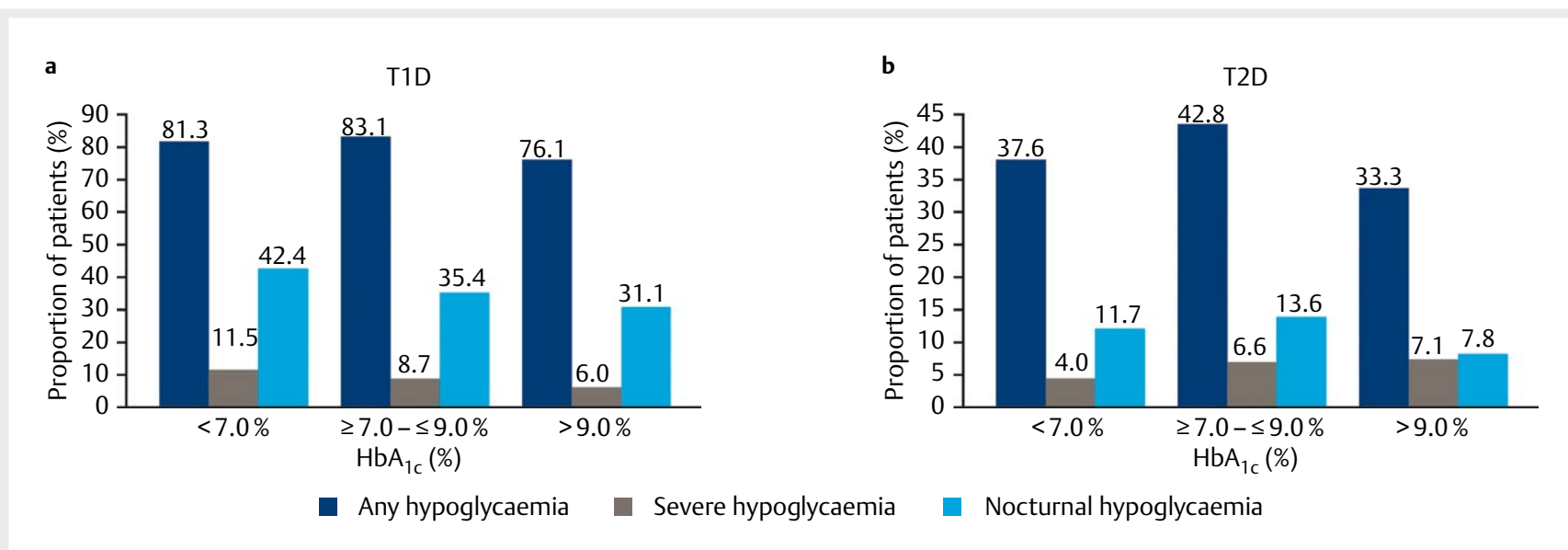

Fig. 2 Proportions of patients with hypoglycaemia by $\mathrm{HbA}_{1 \mathrm{c}}$ level in patients with a type 1 diabetes (T1D) and $\mathbf{b}$ type 2 diabetes (T2D) in the prospective period.

- Table 3 Patient perspectives and actions relating to hypoglycaemia.

\begin{tabular}{|l|c|c|}
\hline & T1D (n=811) & T2D (n=1619) \\
\hline $\begin{array}{l}\text { Knew what hypoglycaemia } \\
\text { was at baseline before } \\
\text { Part 1 SAQ, n/N total (\%) }\end{array}$ & $782 / 804(97.3)$ & $1402 / 1606(87.3)$ \\
\hline \multicolumn{2}{|l|}{ Fear of hypoglycaemia (scale $0-10), \mathbf{n}(\%)$} \\
\hline 0 (Not afraid at all) & $104(13.0)$ & $435(27.4)$ \\
\hline 1 & $66(8.3)$ & $124(7.8)$ \\
\hline 2 & $79(9.9)$ & $189(11.9)$ \\
\hline 3 & $116(14.5)$ & $183(11.5)$ \\
\hline 4 & $74(9.3)$ & $128(8.1)$ \\
\hline 5 & $139(17.4)$ & $211(13.3)$ \\
\hline 6 & $47(5.9)$ & $79(5.0)$ \\
\hline 7 & $60(7.5)$ & $79(5.0)$ \\
\hline 8 & $67(8.4)$ & $73(4.6)$ \\
\hline 9 & $24(3.0)$ & $36(2.3)$ \\
\hline 10 (Absolutely terrified) & $23(2.9)$ & $53(3.3)$ \\
\hline $\begin{array}{l}\text { Increased blood glucose } \\
\text { monitoring as a result of } \\
\text { hypoglycaemia, n (\%) }\end{array}$ & $587(74.3)$ & $780(49.6)$ \\
\hline $\begin{array}{l}\text { SAQ, self-assessment questionnaire; T1D, type 1 diabetes; T2D, type } \\
2 \text { diabetes }\end{array}$ & \\
\hline
\end{tabular}

The results of their evaluation showed that approximately $25 \%$ of the healthcare cost of patients with diabetes was a result of managing hypoglycaemia. The authors suggested specific secondary complications could be avoided by good primary care and management of hyperglycaemia, helping to reduce overall healthcare costs.

In the current analysis of the German cohort of the HAT study, the incidence of hypoglycaemic events requiring hospital admission was higher prospectively. This may be the result of increased patient knowledge of hypoglycaemia as a consequence of participating in the study. Thus patients may have a greater awareness of hypoglycaemia and its complications after completion of the first part of the study.
Limitations of the current study include its observational nature and short prospective period. In addition, as hypoglycaemia is selfreported, results may be confounded due to the subjective nature of the reporting. However, this also represents a strength of the study. Self-reporting of hypoglycaemia captures information where patients may have missed or neglected their blood glucose testing, or were unaware of the threshold at which blood glucose concentration represents a hypoglycaemic event.

In conclusion, the results of this analysis show that the estimated rates of overall, nocturnal and severe hypoglycaemia are higher in this cohort of patients in Germany than those previously reported in other studies, indicating hypoglycaemia may be retrospectively under-reported. Our data highlight the importance of hypoglycaemia when considering individual goals in diabetes therapy and provide important, real-world rates of hypoglycaemic events from Germany.

\section{Acknowledgements}

Medical writing and submission support were provided by Julie Sawyers and Gabrielle Parker of Watermeadow Medical, an Ashfield company, part of UDG Healthcare plc, funded by Novo Nordisk. The authors would also like to thank the practitioners, nurses and study co-ordinators for their help.

\section{Conflicts of Interest}

WK has received honoraria for lectures and for consultancy from Novo Nordisk, MSD, Boehringer Ingelheim, Sanofi-Aventis, Eli Lilly and AstraZeneca. He has also received honoraria for lectures from Amgen, Chiesi, Berlin Chemie, Medtronic, BMS and Servier. AH has no conflicts of interest. CM and JK are employees of Novo Nordisk. MR is managing director of Hamburg MVZ GmbH, which treats patients with diabetes. MR acts as a consultant for the insurance company Deutsche Angestellen Krankenkasse (DAK), television broadcasting company Norddeutscher Rundfunkt (NDR), Astra Zeneca and MSD. Furthermore, MR is a member of the managing committee of the Bundesverband Deutscher Ernährungsmediziner (BDEM). BK is general manager of FIDAM (Forschungsinstitut der Diabetes-Akademie Bad Mergen- 
theim), which is engaged in clinical studies on behalf of various research institutions and companies. BK has acted as a consultant, advisory member for, and received research grants from, Novo Nordisk, Sanofi, Roche, Abbott and Berlin Chemie. Practitioners received a 60 Euro honorarium for their help in enrolling patients to the study.

\section{References}

[1] International Diabetes Federation. IDF Diabetes Atlas, 7th edn. 2016.Available at http://www.diabetesatlas.org (last accessed 22 June 2016).

[2] Barnett AH, Cradock S, Fisher M et al. Key considerations around the risks and consequences of hypoglycaemia in people with type 2 diabetes. Int J Clin Pract. 2010; 64: 1121-1129

[3] Workgroup on Hypoglycemia, American Diabetes Association. Defining and reporting hypoglycemia in diabetes: a report from the American Diabetes Association Workgroup on Hypoglycemia. Diabetes Care. 2005; 28: 1245-1249

[4] Zoungas S, Patel A, Chalmers J et al. ADVANCE Collaborative Group. Severe hypoglycemia and risks of vascular events and death. N Engl J Med. 2010; 363: 1410-1418

[5] Khunti K, Alsifri S, Aronson R et al. HAT Investigator Group. Rates and predictors of hypoglycaemia in 27585 people from 24 countries with insulin-treated type 1 and type 2 diabetes: the global HAT study. Diabetes Obes Metab. 2016; 18: 907-915

[6] Hex N, Bartlett C, Wright D et al. Estimating the current and future costs of Type 1 and Type 2 diabetes in the UK, including direct health costs and indirect societal and productivity costs. Diabet Med 2012; 29: 855-862

[7] Graveling AJ, Deary IJ, Frier BM. Acute hypoglycemia impairs executive cognitive function in adults with and without type 1 diabetes. Diabetes Care. 2013; 36: 3240-3246

[8] Brod M, Wolden M, Christensen T et al. Understanding the economic burden of nonsevere nocturnal hypoglycemic events: impact on work productivity, disease management, and resource utilization. Value Health. 2013; 16: 1140-1149

[9] Geelhoed-Duijvestijn PH, Pedersen-Bjergaard U, Weitgasser R et al. Effects of patient-reported non-severe hypoglycemia on healthcare resource use, work-time loss, and wellbeing in insulin-treated patients with diabetes in seven European countries. J Med Econ 2013; 16: 1453-1461
[10] Östenson CG, Geelhoed-Duijvestijn P, Lahtela J et al. Self-reported nonsevere hypoglycaemic events in Europe. Diabet Med 2014; 31: 92-101

[11] Lüddeke HJ, Sreenan S, Aczel S et al. PREDICTIVE Study Group. PREDICTIVE - a global, prospective observational study to evaluate insulin detemir treatment in types 1 and 2 diabetes: baseline characteristics and predictors of hypoglycaemia from the European cohort. Diabetes Obes Metab. 2007; 9: 428-434

[12] Leiter LA, Boras D, Woo VC. Dosing irregularities and self-treated hypoglycemia in type 2 diabetes: results from the Canadian cohort of an international survey of patients and healthcare professionals. Can J Diabetes 2014; 38: 38-44

[13] Munro N, Barnet AH. Incidence, worry and discussion about dosing irregularities and self-treated hypoglycaemia amongst HCPs and patients with type 2 diabetes: results from the UK cohort of the Global Attitudes of Patient and Physicians (GAPP2) survey. Int J Clin Pract. 2014; 68: 692-699

[14] Pedersen-Bjergaard U, Pramming S, Thorsteinsson B. Recall of severe hypoglycaemia and self-estimated state of awareness in type 1 diabetes. Diabetes Metab Res Rev 2003; 19: 232-240

[15] Elliott L, Fidler C, Ditchfield A et al. Hypoglycemia event rates: a comparison between real-world data and randomized controlled trial populations in insulin-treated diabetes. Diabetes Ther 2016; 7: 45-60

[16] Kulzer B, Seitz L, Kern W. Real-world patient-reported rates of non-severe hypoglycaemic events in Germany. Exp Clin Endocrinol Diabetes. 2014; 122: 167-172

[17] World Medical Association Declaration of Helsinki: ethical principles for medical research involving human subjects. JAMA 2013; 310: 2191-2194

[18] International Conference on Harmonisation. 1996; ICH Harmonised Tripartate Guideline. Good Clinical Practice 1996. Available at: http:// www.ich.org/products/guidelines/efficacy/efficacy-single/article/ good-clinical-practice.html (last accessed 12 April 2017).

[19] Heller SR, Frier BM, Hersløv ML et al. Severe hypoglycaemia in adults with insulin-treated diabetes: impact on healthcare resources. Diabet Med 2016; 33: 471-477

[20] Köster I, Huppertz E, Hauner $\mathrm{H}$ et al. Costs of Diabetes Mellitus (CoDiM) in Germany, direct per-capita costs of managing hyperglycaemia and diabetes complications in 2010 compared to 2001. Exp Clin Endocrinol Diabetes. 2014; 122: 510-516 